\title{
乌s \\ Pile up management at the high-luminosity LHC and introduction to the crab-kissing concept
}

\author{
Stéphane Fartoukh* \\ CERN, CH 1211 Geneva 23, Switzerland \\ (Received 10 June 2014; published 6 November 2014)
}

\begin{abstract}
Upgrading the integrated performance of the LHC, while preserving the quality of the physics data delivered to the experiments, is a real challenge for the high luminosity LHC (HL-LHC). This paper will give an overview of the situation in terms of performance and so-called pile up density which directly impacts on the reconstruction efficiency of the primary vertices at the interaction point. Both the present HL-LHC baseline and its possible extension with the so-called crab-kissing scheme will be discussed in this context.

DOI: 10.1103/PhysRevSTAB.17.111001

PACS numbers: 29.27.-a, 41.85.-p
\end{abstract}

\section{INTRODUCTION AND MOTIVATIONS}

The HL-LHC is being designed to deliver an integrated luminosity of at least $250 \mathrm{fb}^{-1}$ /year in each of the two highluminosity LHC experiments ATLAS and CMS [1], while operating the other two experiments, ALICE and LHCb, at very low and moderate luminosity, of about $10^{31}$ and $1-2 \times 10^{33} \mathrm{~cm}^{-2} \mathrm{~s}^{-1}$, respectively. The ambitious performance target for ATLAS and CMS cannot be met without pushing to the extreme both the optics, namely $\beta^{*}$ [2], and the nominal parameters of the LHC beam [3,4]. It relies as well on a number of very challenging new equipments and key innovative technologies, such as: (i) new larger aperture super-conducting magnets in order to preserve the transverse acceptance of the two high-luminosity insertions at low $\beta^{*}$ $[5,6]$, and (ii) crab-cavities which are high-frequency RF transverse deflectors, ensuring quasi-head-on collisions at the interaction point (IP) despite of the crossing angle, hence preserving the luminosity gain with $1 / \beta^{*}$ (see [7] which introduced the crab-crossing concept, and [8] for proposing it as an ingredient for upgrading the LHC performance).

The instantaneous luminosity is however limited by several factors, in particular the number of interactions per bunch crossing (pile up) which can rapidly degrade the quality of the data collected for the physics analysis. In this respect, the HL-LHC relies on a constant instantaneous luminosity, not exceeding $5 \times 10^{34} \mathrm{~cm}^{-2} \mathrm{~s}^{-1}$ for a $25 \mathrm{~ns}$ bunch spacing, and corresponding to about 140 pile up (PU) events on average per bunch crossing. This is achieved counting on very challenging luminosity leveling techniques, presently via a gradual reduction of $\beta^{*}$ in order to compensate for the proton burn off during the physics store. In order to sustain such a high luminosity, over a typical period of

\footnotetext{
*stephane.fartoukh@cern.ch

Published by the American Physical Society under the terms of the Creative Commons Attribution 3.0 License. Further distribution of this work must maintain attribution to the author(s) and the published article's title, journal citation, and DOI.
}

8-9 hours, the beam parameters, in particular the total beam current, shall correspond to a so-called virtual luminosity which would be about 4 times higher, of the order $2 \times$ $10^{35} \mathrm{~cm}^{-2} \mathrm{~s}^{-1}$ if all the other parameters, for instance $\beta^{*}$, would be pushed to the limits since the very beginning of the leveling process. The baseline HL-LHC parameters (25 ns version [9]) are listed in Table I, including some key quantities such as the virtual luminosity defined above (taking into account the hour glass effect and the RF curvature of the crab-cavity deflecting field), but also other quantities which will be introduced later, such as the peak PU density in time and space domains (taking $85 \mathrm{mb}$ for the inelastic hadron cross-section), the r.m.s. size of the luminous region and the collision time.

TABLE I. Baseline parameters of the HL-LHC (25 ns version [9]) and comparison with the nominal LHC [10]

\begin{tabular}{|c|c|c|}
\hline Parameters & $\begin{array}{c}\text { Nominal } \\
\text { LHC }\end{array}$ & $\begin{array}{l}\text { HL- } \\
\text { LHC }\end{array}$ \\
\hline Energy $[\mathrm{TeV}]$ & 7 & 7 \\
\hline Bunch spacing $[\mathrm{ns}]$ & 25 & 25 \\
\hline Number of bunches & 2808 & 2808 \\
\hline Bunch charge $\left[10^{11}\right]$ & 1.15 & 2.2 \\
\hline Total current $[\mathrm{A}]$ & 0.58 & 1.11 \\
\hline Bunch length $[\mathrm{cm}]$ & 7.50 & 7.50 \\
\hline Energy spread $\left[10^{-4}\right]$ & 1.20 & 1.20 \\
\hline Long. emittance [eVs] & 2.50 & 2.50 \\
\hline$\beta^{*}[\mathrm{~cm}]$ & 55 & 15 \\
\hline Full crossing angle $[\mu \mathrm{rad}]$ & 285 & 590 \\
\hline Beam separation $[\sigma]$ & 9.4 & 12.5 \\
\hline Normalized transverse emittance $[\mu \mathrm{m}]$ & 3.75 & 2.5 \\
\hline Virtual luminosity $\left[10^{34} \mathrm{~cm}^{-2} \mathrm{~s}^{-1}\right]$ & 1.0 & 20.1 \\
\hline Levelled luminosity $\left[10^{34} \mathrm{~cm}^{-2} \mathrm{~s}^{-1}\right]$ & $\ldots$ & 5.0 \\
\hline Levelling time $[\mathrm{h}]$ & $\ldots$ & 8.6 \\
\hline Pile up events/crossing & 27 & 135 \\
\hline Peak line PU density $\left[\mathrm{mm}^{-1}\right]$ & 0.24 & 1.22 \\
\hline Size of the luminous region (r.m.s) $[\mathrm{cm}]$ & 4.5 & 4.4 \\
\hline Peak time PU density $\left[\mathrm{ps}^{-1}\right]$ & 0.06 & 0.32 \\
\hline Collision time (r.m.s.) [ps] & 177 & 163 \\
\hline
\end{tabular}


The next section will derive a series of general formulas for a proper evaluation of the luminosity and corresponding space-time PU density, assuming various possible topologies for the two-dimensional collision footprint. In particular, as part of the HL-LHC baseline, crab-cavities will be assumed to be acting on the beam in the crossing plane ("crab-crossing"), but possibly as well as in the other transverse plane, the so-called parallel separation plane, which forms the novelty of the crab-kissing (CK) scheme. In the baseline scenario, with crab-crossing and luminosity leveling with $\beta^{*}$, Sec. III will investigate possible mitigation measures for the line PU density, acting on the bunch length and/or the bunch shape. A direct comparison with the CK scheme will be given in Sec. IV, while the last section will be devoted to the analysis of the two schemes in terms of leveling techniques and integrated performance.

\section{LUMINOSITY AND PILE UP DENSITY: A COLLECTION OF GENERAL AND SIMPLIFIED FORMULAS}

\section{A. Parametrization of the collision footprint with crossing-angle and crab-cavities}

Let us consider two counter-rotating proton bunches, each of charge $N_{b}$, assumed to be ultrarelativistic, and with a line density $\rho\left(z_{1,2}\right)$ normalized to 1 :

$$
\int d z \rho(z) \equiv 1
$$

The centers of the two bunches, $z_{1}=z_{2}=0$, are assumed to collide at the center of the detector $z=0$ at time $t=0$. Two arbitrary slices $z_{1,2}$ will therefore collide at time $t=-\left(z_{1}+z_{2}\right) /(2 c)$ and at a longitudinal position $z=$ $\left(z_{1}-z_{2}\right) / 2$ inside the detector (with the conventions that $z_{1,2}>0$ corresponds to the bunch head for both beams, and that $z>0$ is oriented according to the direction $z_{1}$ of the first beam). These two slices of charge at $z_{1,2}=-(c t \mp z)$ are assumed to be Gaussian in the two transverse planes, with r.m.s. beam sizes which are given by:

$$
\sigma_{x, y}^{*}(z) \equiv \sqrt{\epsilon \beta_{x, y}^{*}} \sqrt{1+\left(z / \beta_{x, y}^{*}\right)^{2}},
$$

with $\epsilon$ denoting the r.m.s. physical emittance of the beam (assumed to be the same in the horizontal and vertical planes), and taking into account the hour-glass effect at the $z$ position. Assuming these two slices collide with a horizontal and vertical offset, namely $\delta x^{*}(z, t)$ and $\delta y^{*}(z, t)$, respectively, the luminosity of this collision is given by

$$
\begin{aligned}
\delta \mathcal{L}(z, t) & =\frac{N_{b}^{2}}{4 \pi \sigma_{x}^{*}(z) \sigma_{y}^{*}(z)} \exp \left[-\left(\frac{\delta x^{*}(z, t)}{2 \sigma_{x}^{*}(z)}\right)^{2}-\left(\frac{\delta y^{*}(z, t)}{2 \sigma_{y}^{*}(z)}\right)^{2}\right] \rho\left(z_{1}\right) \rho\left(z_{2}\right) d z_{1} d z_{2} \\
& =\frac{2 c N_{b}^{2}}{4 \pi \sigma_{x}^{*}(z) \sigma_{y}^{*}(z)} \exp \left[-\left(\frac{\delta x^{*}(z, t)}{2 \sigma_{x}^{*}(z)}\right)^{2}-\left(\frac{\delta y^{*}(z, t)}{2 \sigma_{y}^{*}(z)}\right)^{2}\right] \rho(-c t+z) \rho(-c t-z) d z d t .
\end{aligned}
$$

With a non-zero crossing angle, e.g., in the horizontal plane, and in the presence of crab-cavities acting on the beams both in the crossing and parallel separation planes, the offsets $\delta x^{*}(z, t)$ and $\delta y^{*}(z, t)$ can be parametrized as follows:

$$
\left\{\begin{array}{l}
\delta x^{*}(z, t)=2 \theta_{\times} z+\frac{\alpha_{\times}}{\omega_{\mathrm{RF}} / c}\left[\sin \left(\omega_{\mathrm{RF}} z_{2} / c+\phi_{\times_{2}}\right)-\sin \left(\omega_{\mathrm{RF}} z_{1} / c+\phi_{\times_{1}}\right)\right] \\
\delta y^{*}(z, t)=\frac{\alpha_{\|}}{\omega_{\mathrm{RF}} / c}\left[\sin \left(\omega_{\mathrm{RF}} z_{2} / c+\phi_{\|_{2}}\right)-\sin \left(\omega_{\mathrm{RF}} z_{1} / c+\phi_{\|_{1}}\right)\right]
\end{array}, \quad \text { with } \quad z_{1,2} \equiv-(c t \mp z),\right.
$$

where $\theta_{\times}$denotes the half crossing angle (see Fig. 1), the quantities $\alpha_{\times, \|}$represent the beam rotation angles induced by the crab-cavities in the crossing and parallel separation planes, respectively, and the phase lags $\phi_{\times_{1,2}, \|_{1,2}}$ are adjusted in the following way. In all the rest of the paper, the crab-cavities will be assumed to be in phase in the crossing plane, i.e., $\phi_{\times_{1,2}} \equiv 0$, and in antiphase in the parallel separation plane, i.e., $\phi_{\|_{1}} \equiv 0$ and $\phi_{\|_{2}} \equiv \pi$, such that

$$
\left\{\begin{array}{l}
\delta x^{*}(z, t)=2\left[\theta_{\times} z-\frac{\alpha_{\times}}{\omega_{\mathrm{RF}} / c} \cos \left(\omega_{\mathrm{RF}} t\right) \sin \left(\omega_{\mathrm{RF}} z / c\right)\right] \\
\delta y^{*}(z, t)=2 \frac{\alpha_{\|}}{\omega_{\mathrm{RF}} / c} \sin \left(\omega_{\mathrm{RF}} t\right) \cos \left(\omega_{\mathrm{RF}} z / c\right)
\end{array} .\right.
$$

Strictly speaking, crab-crossing and crab-kissing could also be combined in the crossing plane, namely: (i) by restoring a configuration of head-on collision in the parallel separation plane [i.e., $\alpha_{\|} \equiv 0$ in Fig. 1(b)], and (ii) by increasing the crabbing angle of one of the two beams while decreasing it by the same amount for the other beam [i.e., $\pm \alpha_{\times} \longrightarrow \pm\left(\alpha_{\times} \pm \alpha_{\|}\right)$in Fig. 1(a)]. Such a configuration would therefore result into a net asymmetry between the two beams at the beginning of the luminosity leveling process, in particular with a very high RF deflecting voltage to be applied in the crossing plane for one of the two beams [typically doubled with respect to a full crabbing 


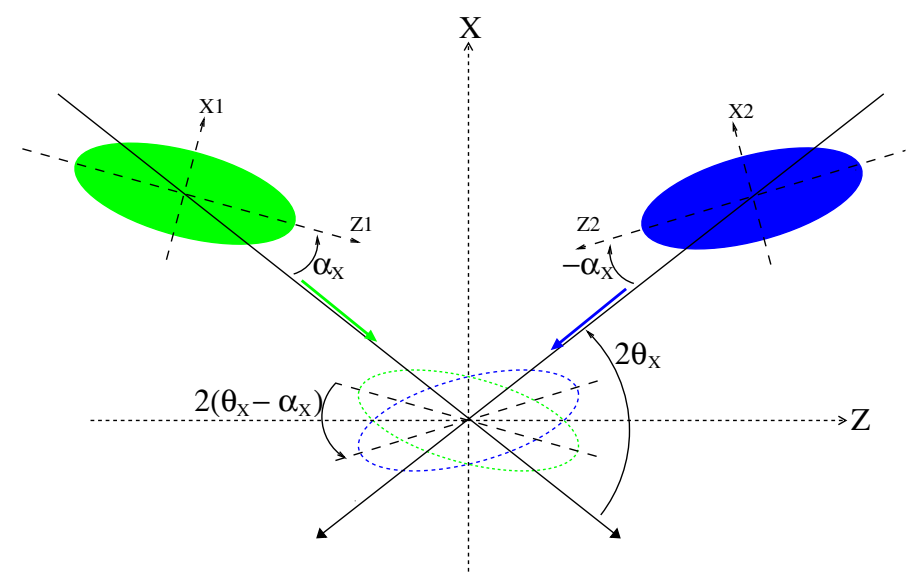

(a)

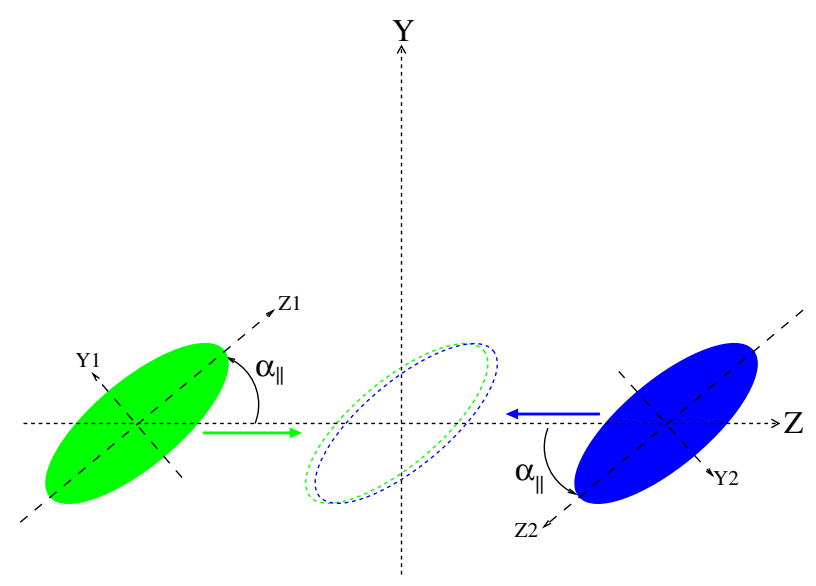

(b)

FIG. 1. Sketch of the four-dimensional collision footprint in the presence of a crossing angle, and with crab-cavities acting both in the crossing plane [crab-crossing configuration showed in (a)] and in the other transverse plane, the so-called parallel separation plane [crabkissing configuration showed in (b)]. The hour-glass effect and the RF curvature of the crab-cavity deflecting field are not taken into account in these illustrations.

configuration corresponding to $\theta_{\times} \equiv \alpha_{\times}$in Fig. 1(a)], and an almost vanishing voltage for the other beam. On the other hand, one advantage, at least a priori, would be the development of only one type of structure and cryomodule, i.e., for horizontal only or vertical only RF deflections. However, the HL-LHC needs both types anyway in order to cope with an alternated HV crossing scheme, i.e., horizontal and then vertical, in the two high luminosity insertions of the ring. A more symmetric layout is then more favorable for the HL-LHC, i.e., with each of the two insertions equipped with both horizontal and vertical crab-cavities and the collision configuration illustrated in Fig. 1. Therefore a possible variant with crab-kissing collisions in the crossing plane will no longer be discussed in all the rest of paper, although the following developments could be easily reformulated in order to study as well this configuration.

\section{B. General expressions for the luminosity and several pile up related quantities}

Combining (2), (3), and (5), the two-dimensional density of luminosity can therefore be written as follows:

$$
\frac{\partial^{2} \mathcal{L}}{\partial z \partial t}=\mathcal{L}_{0} \times 2 c \frac{K(z, t)}{R} \rho(c t+z) \rho(c t-z),
$$

where $\mathcal{L}_{0}$ is the total luminosity integrated over the collision of the two bunches

$$
\mathcal{L}_{0} \equiv \iint d z d t \frac{\partial^{2} \mathcal{L}}{\partial z \partial t} \equiv \frac{N_{b}^{2}}{4 \pi \epsilon \sqrt{\beta_{\times}^{*} \beta_{\|}^{*}}} \times R,
$$

$R$ represents the generalized luminosity loss factor given by

$$
R \equiv 2 c \iint d z d t K(z, t) \rho(c t+z) \rho(c t-z),
$$

and the kernel function $K(z, t)$ is defined by

$$
K(z, t) \equiv \frac{\exp \left\{-\frac{\left[\theta_{\times} z-\frac{\alpha_{\times}}{\omega_{\mathrm{RF}} / c} \cos \left(\omega_{\mathrm{RF}} t\right) \sin \left(\omega_{\mathrm{RF}} z / c\right)\right]^{2}}{\epsilon \beta_{\times}^{*}\left[1+\left(z / \beta_{\times}^{*}\right)^{2}\right]}\right\}}{\sqrt{1+\left(z / \beta_{\times}^{*}\right)^{2}} \sqrt{1+\left(z / \beta_{\|}^{*}\right)^{2}}} \times \exp \left\{-\frac{\left[\frac{\alpha_{\|}}{\omega_{\mathrm{RF}} / c} \sin \left(\omega_{\mathrm{RF}} t\right) \cos \left(\omega_{\mathrm{RF}} z / c\right)\right]^{2}}{\epsilon \beta_{\|}^{*}\left[1+\left(z / \beta_{\|}^{*}\right)^{2}\right]}\right\},
$$

with $\beta_{\times}^{*}$ and $\beta_{\|}^{*}$ representing $\beta^{*}$ in the crossing and parallel separation planes, respectively. From Eq. (6), follows immediately the expression for the 2D PU density:

$$
\frac{\partial^{2} \mu}{\partial z \partial t}(z, t)=\mu_{\mathrm{tot}} \times 2 c \frac{K(z, t)}{R} \rho(c t+z) \rho(c t-z),
$$

with $\mu_{\text {tot }} \equiv \sigma \mathcal{L}_{0}$ denoting the total number of interactions produced on average in the collision ( $\sigma$ being the inelastic hadron cross-section). Finally the line and time PU densities are given by

$$
\mu_{z}(z) \equiv \int d t \frac{\partial^{2} \mu}{\partial z \partial t} \quad \text { and } \quad \mu_{t}(t) \equiv \int d z \frac{\partial^{2} \mu}{\partial z \partial t}
$$


and the r.m.s. length of the luminous region and the collision time can be calculated with the following $2 \mathrm{D}$ integrals:

$$
\begin{aligned}
& \sigma_{z, \operatorname{lum}}^{2}=\frac{1}{\mu_{\mathrm{tot}}} \times \iint d z d t z^{2} \frac{\partial^{2} \mu}{\partial z \partial t} \text { and } \\
& \sigma_{t, \operatorname{lum}}^{2}=\frac{1}{\mu_{\mathrm{tot}}} \times \iint d z d t t^{2} \frac{\partial^{2} \mu}{\partial z \partial t} .
\end{aligned}
$$

\section{Approximations and discussion}

Neglecting the hour-glass effect and the RF curvature of the crab-cavity deflecting field [i.e., $z / \beta^{*} \sim 0, \cos (x) \sim 1$ and $\sin (x) \sim x$ in Eq. (9)], the expression for the kernel function $K(z, t)$ can be reduced to

$$
K(z, t) \approx \exp \left[-\left(\tilde{\theta}_{\times}-\tilde{\alpha}_{\times}\right)^{2} \frac{z^{2}}{\beta_{\times}^{2}}\right] \times \exp \left[-\left(\tilde{\alpha}_{\|}\right)^{2} \frac{(c t)^{2}}{\beta_{\|}^{2}}\right],
$$

where the quantities with a tilde keep the same meaning as before but are normalized by the beam divergence at the IP, namely $\tilde{\theta}_{\times} \equiv \theta_{\times} / \sqrt{\epsilon / \beta_{\times}^{*}}, \tilde{\alpha}_{\times} \equiv \alpha_{\times} / \sqrt{\epsilon / \beta_{\times}^{*}}$ and $\tilde{\alpha}_{\|} \equiv \alpha_{\|} / \sqrt{\epsilon / \beta_{\|}^{*}}$. Using the above approximation, analytical expressions can then be derived for several quantities, such as the line and time PU densities, and for the generalized luminosity loss factor $R$. These expressions are reported in Table II in the two cases of a Gaussian and a rectangular bunch distribution, together with the introduction of key physical quantities, either well known such as the Piwinsky angle, or new ones, such as the so-called time Piwinsky angle.

By inspecting these various expressions, the duality between line and time PU density becomes obvious via the exchange between the two Piwinsky angles $\phi$ (standard) and $\psi$ ("time" Piwinsky angle). In the case of Gaussian bunches, acting only on the crab-cavities in the crossing-plane to level the luminosity, i.e., increasing the Piwinsky angle $\phi$ at $\psi \equiv 0$ (no kissing), increases the peak line PU density, i.e., shortens the luminous region, but does not impact on the time PU density. On the other hand, acting on the time Piwinsky angle $\psi$ at $\phi=0$ (full crabbing in the crossing plane and crab-kissing in the parallel separation plane) reduces the collision time but does not impact on the luminous region. This duality then leads to even more interesting results in the case of rectangular bunches. At $\phi=\psi=0$ (full crabbing, no kissing) it is easy to see that the line PU density becomes triangular $(\operatorname{Erf}(\psi u) / \psi \approx 2 u / \sqrt{\pi}$ at small $\psi)$, as expected from the convolution product of two rectangular distributions. Although less obvious, the situation is in fact exactly the same for the time PU density, as one may deduce by evaluating the expressions given in Table II in the limit $\phi=\psi=0$, or more simply by using the duality principle mentioned above. A more intuitive result is the time PU density becoming flatter and flatter at $\psi=0$ (no kissing) and large Piwinsky angle $\phi$, as could be deduced from Table II $(\operatorname{Erf}(|u|) \approx 1$ for $u \gg 1)$, or simply inferred from the time invariant configuration of two coasting beams colliding with an angle. Then, the duality principle described above imposes the line PU density to become rather flat as well, but at $\phi=0$ (full crabbing) and large time Piwinsky angle $\psi$ (crab-kissing). This turns out to be exactly the case in view of the analytical expression given in this case in Table II.

More quantitatively, in the case of Gaussian bunches, full crabbing $(\phi=0)$, and in the presence or not of crab-kissing (arbitrary $\psi$ ), the peak line PU density can be directly extracted from Table II and is given by

$$
\frac{\partial \mu_{G}}{\partial z}(z=0)=\frac{\mu_{\mathrm{tot}}}{\sqrt{\pi} \sigma_{z}} \approx 0.56 \times \frac{\mu_{\mathrm{tot}}}{\sigma_{z}} \quad \text { at } \phi=0 .
$$

In the same conditions, the generalized luminosity loss factor reads

TABLE II. Simplified expressions for the line and time pile up densities in the case of a Gaussian or rectangular bunch distributions

\begin{tabular}{lccc}
\hline \hline Quantities & Notation & Gaussian & Rectangular (Heaviside step function $\Theta)$ \\
\hline Distribution & $\rho(z)$ & $\frac{1}{\sqrt{2 \pi} \sigma_{z}} e^{-\frac{1}{2}\left(\frac{z}{\sigma_{z}}\right)^{2}}$ & $\frac{1}{2 L} \Theta(1-|z| / L)$ \\
Bunch length r.m.s. & $\sigma_{z}$ & $\sigma_{z}$ & $L / \sqrt{3}$ \\
Standard Piwinsky angle & $\phi$ & $\frac{\left|\tilde{\theta}_{x}-\tilde{\alpha}_{\times}\right| \sigma_{z}}{\beta_{\times}^{*}}$ & $\frac{\left|\tilde{\theta}_{\times}-\tilde{\alpha}_{x}\right| L}{\beta_{\times}^{*}}$ \\
“Time” Piwinsky angle & $\psi$ & $\left|\tilde{\alpha}_{\|}\right| \frac{\sigma_{z}}{\beta_{\|}^{*}}$ & $\left|\tilde{\alpha}_{\|}\right| \frac{L}{\beta_{\|}^{*}}$ \\
Generalized Loss Factor & $\mathrm{R}$ & $\frac{1}{\sqrt{1+\phi^{2}}} \sqrt{1+\psi^{2}}$ & $\frac{\sqrt{\pi}}{L \psi} \int_{0}^{L} d z \operatorname{Erf}[\psi(1-z / L)] e^{-\left(\frac{\phi z}{L}\right)^{2}}$ \\
Line PU density & $\frac{1}{\mu_{\mathrm{tot}}} \frac{\partial \mu}{\partial z}$ & $\frac{\sqrt{1+\phi^{2}}}{\sqrt{\pi} \sigma_{z}} e^{-\left(1+\phi^{2}\right)\left(\frac{z}{z_{z}}\right)^{2}}$ & $\frac{\sqrt{\pi}}{2 R L \psi} \operatorname{Erf}[\psi(1-|z| / L)] \Theta(1-|z| / L) e^{-\left(\frac{\phi z}{L}\right)^{2}}$ \\
Time PU density & $\frac{1}{\mu_{\mathrm{tot}}} \frac{\partial \mu}{\partial(c t)}$ & $\frac{\sqrt{1+\psi^{2}}}{\sqrt{\pi} \sigma_{z}} e^{-\left(1+\psi^{2}\right)\left(\frac{c t}{\sigma_{z}}\right)^{2}}$ & $\frac{\sqrt{\pi}}{2 R L \phi} \operatorname{Erf}[\phi(1-|c t| / L)] \Theta(1-|c t| / L) e^{-\left(\frac{\psi c t}{L}\right)^{2}}$ \\
\hline \hline
\end{tabular}




$$
R=\frac{1}{\sqrt{1+\psi^{2}}}<1 \quad \text { at } \phi=0 .
$$

Therefore, the CK scheme offers a very attractive tool for leveling the luminosity, at least at constant line PU density for Gaussian bunches (which would not be the case by acting on the standard Piwinsky angle $\phi$, instead of $\psi$ ). The situation is even more interesting in the case of rectangular bunches, leading in the same conditions to a peak line PU density of the order of

$$
\begin{aligned}
\frac{\partial \mu_{R}}{\partial z}(z=0) & =\frac{\sqrt{\pi} \mu_{\text {tot }}}{2 R L \psi} \operatorname{Erf}(\psi) \stackrel{\psi \gg 1}{\approx} \frac{1}{2 \sqrt{3}} \times \frac{\mu_{\text {tot }}}{\sigma_{z}} \approx 0.29 \times \frac{\mu_{\text {tot }}}{\sigma_{z}} \\
\text { at } \phi & =0 \quad \text { and } \psi \gg 1,
\end{aligned}
$$

where, according to Table II, we have used the asymptotic expression $R \approx \sqrt{\pi} / \psi$ for the loss factor at high $\psi$ and $\phi=0$. This peak density is then reduced by almost a factor of 2 with respect to the case of Gaussian bunches. Finally it is also instructive to assess the situation assuming a rectangular distribution with full crabbing but no crabkissing $(\psi=\phi=0)$. In this case, following the expressions given in Table II and after some algebra, the loss factor is equal to unity, and the line PU distribution is triangular with a peak density given by:

$$
\begin{gathered}
\frac{\partial \mu_{R}}{\partial z}(z=0)=\frac{\mu_{\mathrm{tot}}}{L}=\frac{1}{\sqrt{3}} \times \frac{\mu_{\mathrm{tot}}}{\sigma_{z}} \approx 0.58 \times \frac{\mu_{\mathrm{tot}}}{\sigma_{z}} \\
\text { at } \phi=\psi=0,
\end{gathered}
$$

which is very similar to, and in fact slightly higher than, the peak density obtained in the Gaussian case.

In summary, the CK scheme not only opens the possibility to level the luminosity at constant luminous region by acting on the crab-cavities in the parallel separation plane, but it can also lead to a reduction of the peak line PU density by up to a factor of 2 assuming more uniform bunch distributions. This gain by a factor of 2 in terms of data quality may then be used, fully or partly, to further push the maximum number of interactions allowed on average per bunch crossing, namely $\mu_{\text {tot }}$, therefore the maximum allowed instantaneous luminosity and in fine the integrated performance of the machine.

\section{THE BASELINE SCHEME WITH CRAB-CROSSING AND $\beta^{*}$ LEVELING}

The main disadvantage of the CK scheme is however its cost in terms of new hardware. First of all additional crabcavities are needed for rotating the beam as well in the parallel separation plane (or, in order to work at constant number of crab-cavity modules, assuming more voltage available per cavity, and/or higher beta functions at the crab-cavities, and/or assuming flat $\beta^{*}$ optics with eventually so-called long-range beam-beam wire compensator [11-15] to reduce the crossing angle needed in this case, see later in Sec. IV). Then, in order to produce more uniform longitudinal bunch profiles, a double harmonic RF system working in bunch lengthening mode would be needed, which was already kept as an option for other purposes in the HL-LHC, but would definitely help to fully exploit the potential of the CK concept. Therefore before implementing the CK scheme into the HL-LHC baseline, immediate mitigation measures shall first be investigated, namely acting on the bunch length and/or the bunch profile, and assuming a luminosity leveling scenario with $\beta^{*}$ as in the present HL-LHC running scenario.

\section{A. Performance vs bunch length and bunch profile}

One key ingredient to push the integrated performance of the machine is the ability to maximize the leveling time, namely $T_{\text {lev }}$, during which the instantaneous luminosity can be sustained at its maximum allowed value, presently $\mathcal{L}_{\text {lev }}=5 \times 10^{34} \mathrm{~cm}^{-2} \mathrm{~s}^{-1}$. Assuming marginal emittance growth during this process, and for any luminosity leveling technique, the leveling time is directly given by the ratio $k=\mathcal{L}_{\text {virt }} / \mathcal{L}_{\text {lev }}$ between the virtual luminosity as defined in Sec. I and the leveled luminosity [16]:

$$
T_{\text {lev }}=\left(1-\frac{1}{\sqrt{k}}\right) \times \underbrace{\frac{N_{\text {tot }}}{2 \sigma_{\text {tot }} \mathcal{L}_{\text {lev }}}}_{\equiv T_{\text {beam }}},
$$

where $T_{\text {beam }}$ corresponds to the actual beam life time at the leveled luminosity $\mathcal{L}_{\text {lev }}$, considering the total hadron cross section $\sigma_{\text {tot }}\left(\sigma_{\text {tot }} \approx 100 \mathrm{mb}\right)$, two high luminosity insertions, and a total number of circulating protons $N_{\text {tot }}$ at the beginning of the store. Keeping the geometric loss factor $R$ as close as possible to 1 for the minimum possible $\beta^{*}$, i.e., maximizing the virtual luminosity at constant current, is therefore very important in order to preserve the leveling time, and then the integrated performance per fill. The sensitivity of this parameter with respect to the r.m.s. bunch length $\sigma_{z}$ and to the bunch profile (Gaussian or flat) is showed in Fig. 2, using the relations (8) and (9) with the HL-LHC parameters given in Table I, and assuming $400 \mathrm{MHz}$ crab-cavities with "full-crabbing" in the crossing plane and "no kissing" in the other plane, i.e., $\alpha_{\times} \equiv \theta_{\times}$and $\alpha_{\|} \equiv 0$ in Eq. (9). Depending on whether the machine is equipped with a single- or a double-harmonic RF system working in bunch lengthening mode, the bunch profile was assumed to be purely Gaussian in the first case, and was approximated by the following pseudorectangular distribution in the second case:

$$
\rho\left(z ; \sigma_{z}\right)=\frac{2^{\frac{5}{4}} \sqrt{\pi}}{\left[\Gamma\left(\frac{1}{4}\right)\right]^{2} \sigma_{z}} \exp \left[-\frac{1}{2}\left(\frac{z}{\Gamma\left(\frac{1}{4}\right) / \sqrt{2 \pi} \sigma_{z}}\right)^{4}\right],
$$




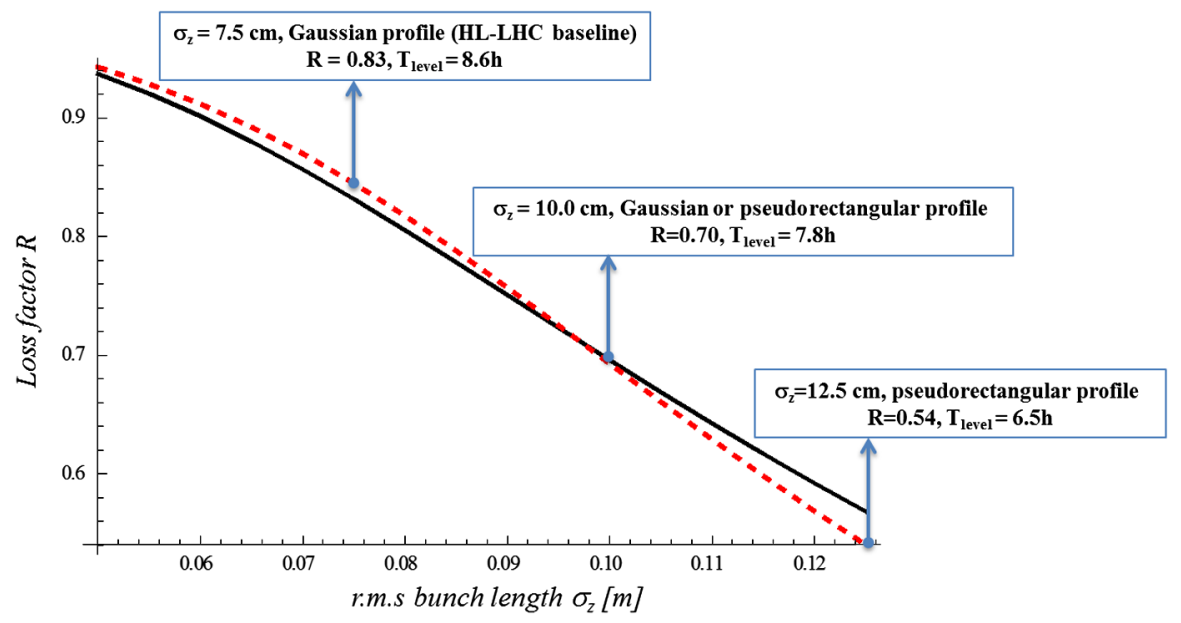

FIG. 2. Generalized luminosity loss factor $R$ [see Eq. (8)] at the end of the luminosity leveling process (full crabbing, no crab-kissing, $\beta^{*}=15 \mathrm{~cm}$ ) as a function of the r.m.s. bunch length $\sigma_{z}[\mathrm{~m}]$, including the hour-glass effect, considering the RF curvature of the crabcavity deflecting field, and using the HL-LHC parameter set given in Table I (except for the bunch length). The solid line assumes a purely Gaussian bunch profile (single harmonic RF system), while the dashed curve corresponds to the case of a double harmonic RF system, which is simulated by considering the pseudorectangular distribution parametrized in Eq. (19).

which is normalized to 1 , and parametrized such that its standard deviation is precisely $\sigma_{z}$. For the range of bunch length of interest, i.e., in between $7.5 \mathrm{~cm}$ and $12.5 \mathrm{~cm}$ r.m.s., the situation is very similar in the two cases. Beyond a r.m.s bunch length of $7-8 \mathrm{~cm}$, the luminosity loss factor drops rapidly, not only due to the hour glass effect at $\beta^{*}=$ $15 \mathrm{~cm}$ but also due the RF curvature of the crab-cavity deflecting field which makes the compensation of the crossing angle no longer efficient for the particles in the head and tail of the bunch, namely located at $z \sim \pm \lambda_{400 \mathrm{MHz}} / 8-9 \mathrm{~cm}$ and beyond (halfway in between the zero and peak of the sine RF kick). A r.m.s bunch length of $10 \mathrm{~cm}$ corresponds to a sort of threshold, not only because it hits the capture limit of a single harmonic RF system running at $400 \mathrm{MHz}$, but also because it leads to a reduction of the leveling time above a significance level of lets say $10 \%$, from $8.6 \mathrm{~h}$ to $7.8 \mathrm{~h}$ (see Fig. 2), corresponding to a loss of integrated performance by $1 \%$ on average per fill of $8.6 \mathrm{~h}$.

\section{B. Line pile up density vs bunch length and bunch profile}

In addition, regardless of the bunch profile, a r.m.s. bunch length of $10 \mathrm{~cm}$ brings the peak line PU density close to its minimum at the baseline $\beta^{*}$ of $15 \mathrm{~cm}$ (see Fig. 3). Indeed, working at constant $\mu_{\text {tot }}$ and instead of an intuitive scaling with $1 / \sigma_{z}$, the peak line PU density saturates or can even reincrease at low $\beta^{*}$ and for too long bunches, again due to the hour-glass effect and due the RF curvature of the crab-cavity deflecting field. At this optimal bunch length, the gain in line PU density is however quite modest, in between $20 \%$ at $\beta^{*}=70 \mathrm{~cm}$ (i.e., at the beginning of the store), down to only $10 \%$ at $\beta^{*}=15 \mathrm{~cm}$ (see Fig. 4), from $1.22 \mathrm{event} / \mathrm{mm}$ at $\sigma_{z}=7,5 \mathrm{~cm}$ [Fig. 4(a)] down to about
1.09 event/mm, assuming Gaussian bunch profile with an r.m.s. length pushed up to $\sigma_{z} \approx 10 \mathrm{~cm} \mathrm{r.m.s} \mathrm{[Fig.} \mathrm{4(b)],} \mathrm{i.e.,}$ at the capture limit by the existing $400 \mathrm{MHz}$ RF system of the LHC. Then a few percents but not more can be gained assuming a pseudorectangular bunch profile [Fig. 4(c)].

As already announced, the CK scheme offers a net gain by a factor of 2 and, more precisely, the possibility to flatten the line PU density below 0.6 event $/ \mathrm{mm}$ over the full luminous region. As we will see in the next sections, this net gain is however not only given by the new hardware assumed by the CK scheme (additional crab-cavities acting in the parallel separation plane, double harmonic RF system), but also due to the new concept it introduces,

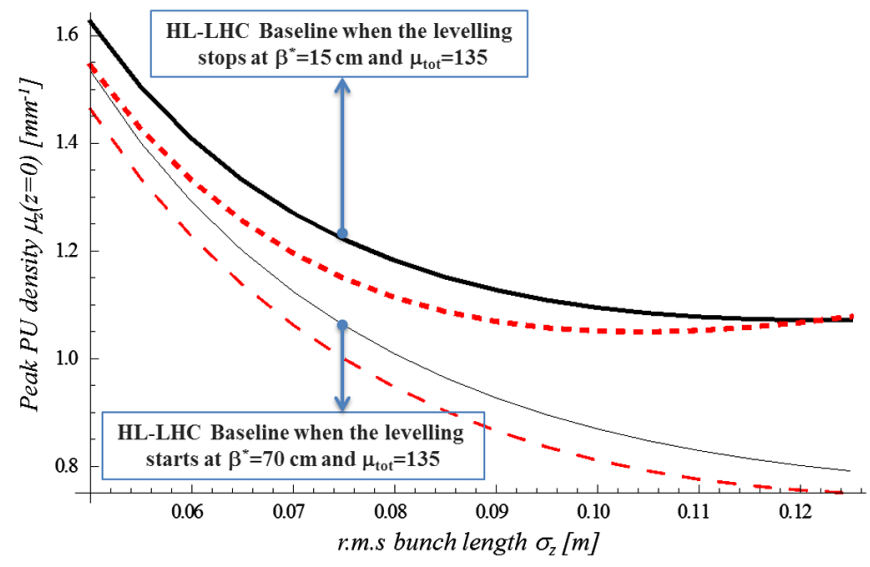

FIG. 3. Peak line PU density $\mu_{z}(z=0)\left[\mathrm{mm}^{-1}\right]$ at $\mu_{\text {tot }}=135$ as a function of the r.m.s bunch length $\sigma_{z}[\mathrm{~m}]$, assuming Gaussian (black solid lines) or pseudorectangular (red dashed lines) bunch profiles at the beginning (thin lines) and in the end (thick lines) of the $\beta^{*}$ leveling process (corresponding to $\beta^{*}=70 \mathrm{~cm}$ and $15 \mathrm{~cm}$, respectively). 


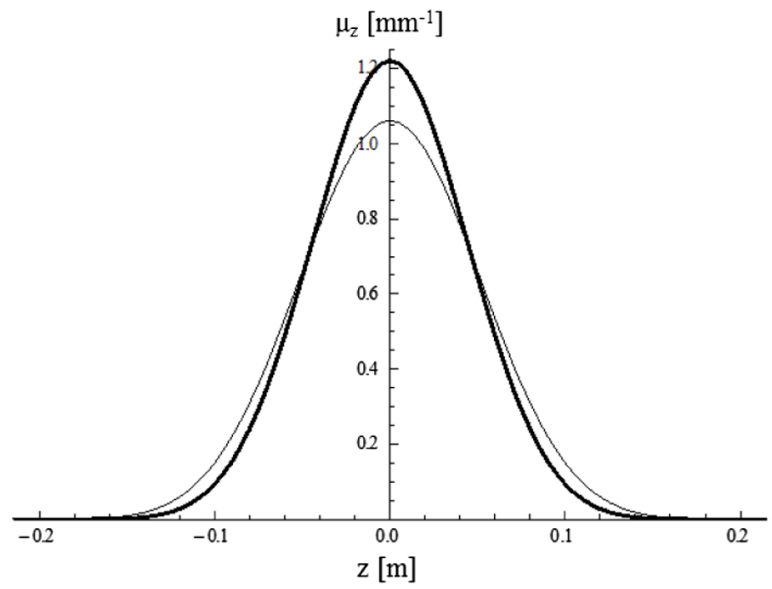

(a)

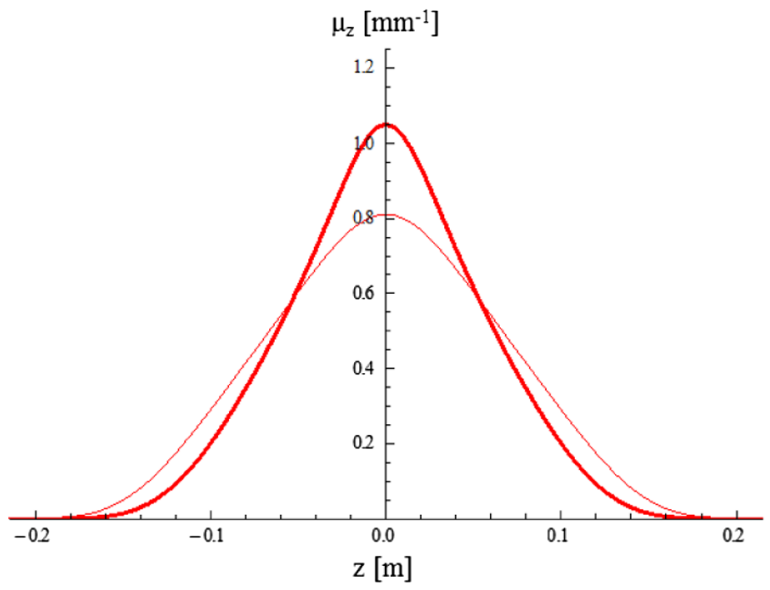

(c)

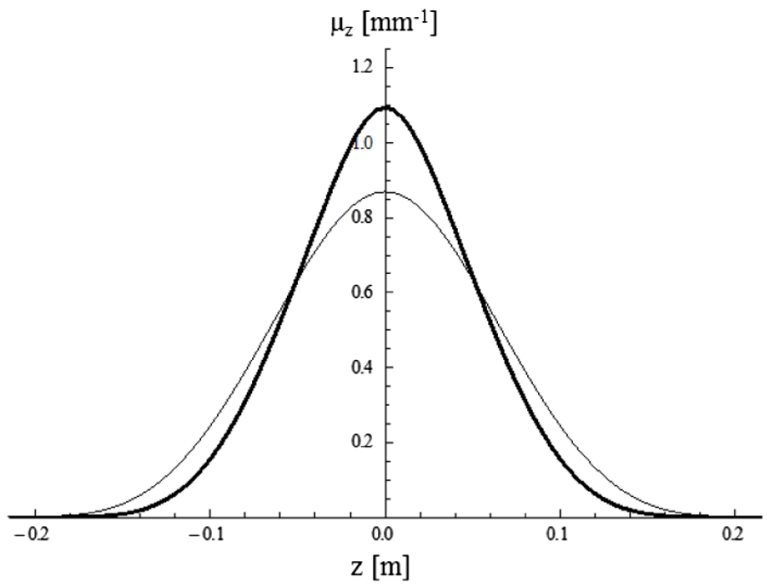

(b)

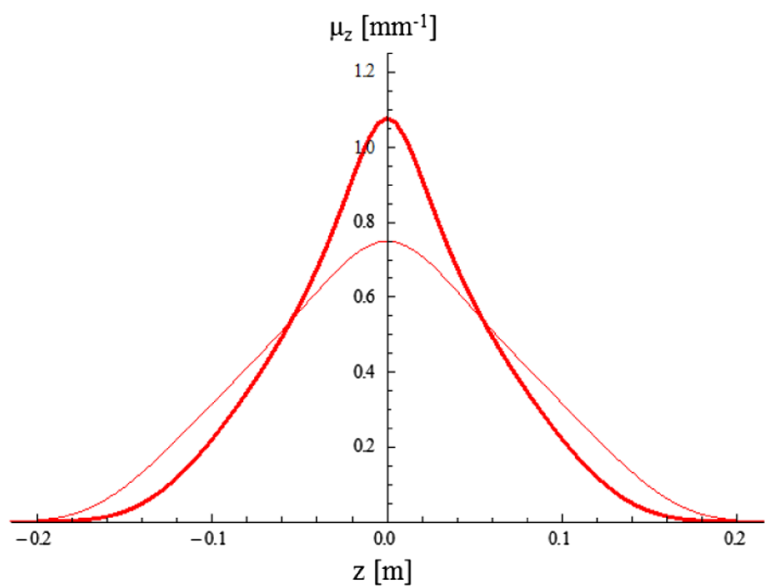

(d)

FIG. 4. Line PU density $\mu_{z}(z)\left[\mathrm{mm}^{-1}\right]$ as a function of the longitudinal position $z[\mathrm{~m}]$ inside the detector. These snapshots are taken at the beginning (thin lines) and in the end (thick lines) of the $\beta^{*}$ leveling process, for different bunch profiles and r.m.s. bunch length $\sigma_{z}$. All these distributions are normalized to $\mu_{\text {tot }}=135$, which represents the total number of interactions per bunch crossing at the leveled luminosity of $5 \times 10^{34} \mathrm{~cm}^{-2} \mathrm{~s}^{-1}$ (see Table I). (a) Gaussian bunch profile with $\sigma_{z}=7.5 \mathrm{~cm}$, (b) Gaussian bunch profile with $\sigma_{z}=10 \mathrm{~cm}$, (c) Pseudo-rectangular bunch profile with $\sigma_{z}=10 \mathrm{~cm}$, and (d) Pseudo-rectangular bunch profile with $\sigma_{z}=12.5 \mathrm{~cm}$.

which is a leveling of the peak line PU density rather than the luminosity itself. It is worth noting that this new leveling concept has already been tested for the HLLHC baseline, namely squeezing $\beta^{*}$ during the store at constant peak PU density, and therefore at gradually reduced instantaneous luminosity (due to the natural degradation of the peak PU density with $\beta^{*}$ at constant luminosity, see Fig. 3). In this case, compromising on a loss of integrated performance of a few percents, the peak line PU density could however not be reduced below $0.9-1.0$ event $/ \mathrm{mm}$, assuming as well a double harmonic RF system and an increase of the r.m.s. bunch length up to $10 \mathrm{~cm}$ in order to reach the lowest value of 0.9 event $/ \mathrm{mm}$ [17]. In summary, a peak line PU density of about 1 event $/ \mathrm{mm}$ seems to be an hard limit for the present HL-LHC baseline and variants, even relying on longer bunches, flatter bunch profiles and/or a sophistication of the leveling scenario with $\beta^{*}$.

\section{THE CRAB-KISSING SCHEME}

In this section, the longitudinal bunch distribution is assumed to be pseudorectangular [see Eq. (19)]. The machine is equipped with two different sets of crab-cavities per beam, acting separately in the crossing and the parallel separation plane. In each of the two transverse planes, the crab-cavities acting on the first and on the second beam are assumed to be synchronized in order to establish the collision configuration illustrated in Fig. 1.

\section{A. The physical understanding of the CK scheme and its natural association with flat optics}

As soon as a sufficient "kissing" angle is introduced in the parallel separation plane (noted $\alpha_{\|}$in Fig. 1), the head, the center and the tail of the first beam only collide with the tail, the center and the head, respectively, of the second beam. These collisions take place almost simultaneously, 
contrary to standard head-on collisions over a finite bunch length. Furthermore, since the charge density is assumed to be more or less the same at the head, the tail and the center of the bunch, the number of interactions is expected to be rather invariant along the luminous region, which also means a reduction of the peak line PU density at a given running luminosity. Using another language, the CK scheme offers a tool to level the collision time, and therefore the luminosity at reduced line pile up density, but also at reduced head-on beam-beam tune shift since the interaction time has shrunk. This is another beneficial sideeffect of the CK scheme, which will however not be analyzed in much more detail in the following.

Finally the thinner the beam in the parallel separation plane, the more important is the impact of a given "kissing angle" on the collision time (which explains the terminology of "Time Piwinsky angle" introduced in Table II), up to a point where infinitely flat beams could even cross without interacting with each other in the physical space. This is one of the two reasons why flat optics (with the plane of highest $\beta^{*}$ corresponding to the crossing plane) are very favorable for the CK scheme, with, for the same effect in terms of PU density, less voltage needed for the crabcavities acting in the parallel separation plane [see also the second terms on the right-hand side of Eq. (9)]:

$$
\alpha_{\|} \propto \sqrt{\beta_{\|}^{*}}
$$

The second reason is that, at constant product $\beta_{\times}^{*} \times \beta_{\|}^{*}$, i.e., constant virtual luminosity, the crossing angle, and therefore the crabbing angle $\alpha_{\times}$needed to preserve the head-on collisions in the crossing plane, could in principle be reduced for flat optics, and even strongly assuming the implementation of electromagnetic wires for compensating the long-range beam-beam interactions [11]:

$$
\alpha_{\times} \propto \theta_{\times} \propto 1 / \sqrt{\beta_{\times}^{*}} \propto \sqrt{\beta_{\|}^{*}} \text { at constant } \beta_{\times}^{*} \times \beta_{\|}^{*} .
$$

\section{B. Possible CK configurations for the HL-LHC and their requirement in terms of crab-cavity voltage}

The possibility of flat optics has therefore been integrated in Table III, where a few possible HL-LHC configurations have been down-selected and analyzed in terms of peak line PU density and crab-cavity voltage requirements. As already discussed for the baseline crabcrossing scenario with round optics (Case a), the peak PU density reaches its maximum in the end of the leveling process with $\beta^{*}$, and this maximum has been reported accordingly in Table III. A net gain by a factor of 2, already at the beginning of the store, then preserved all along, is actually within reach for the three other cases analyzed where the CK scheme has been implemented (see also the next section for a detailed description of the leveling process and techniques, and the performance reach in the case of the CK scheme). Furthermore, as shown in Fig. 5, the three cases studied for crab-kissing are in fact extremely similar in terms of line PU density, while the optics, crossing angle and/or crab-cavity usage are very different in these three cases. In particular a zoom of the line PU density around $z=0$ shows that a density of $0.61 \mathrm{event} / \mathrm{mm}$ reached at the center of the luminous region is probably a minimum for the CK scheme, assuming an r.m.s. bunch length not exceeding $10 \mathrm{~cm}$ and a total number of interactions of $\mu_{\text {tot }}=135$ per bunch crossing. Below this minimum two bumps of density starts clearly to

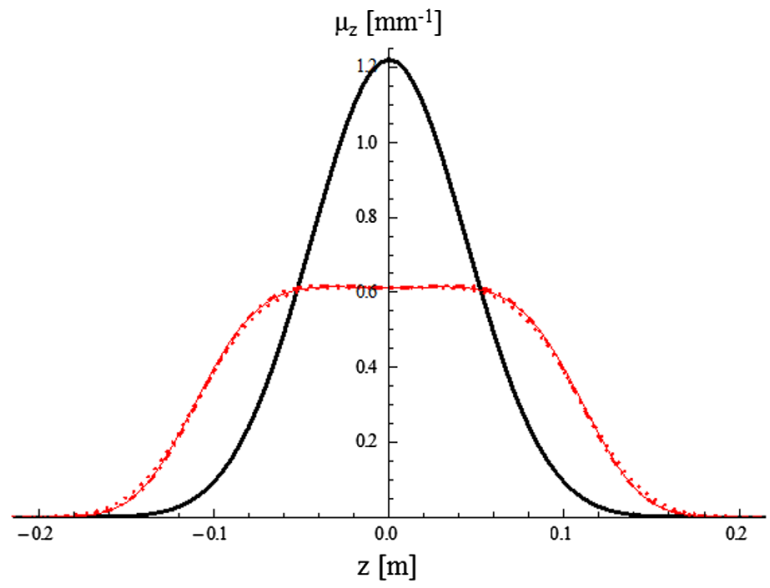

(a)

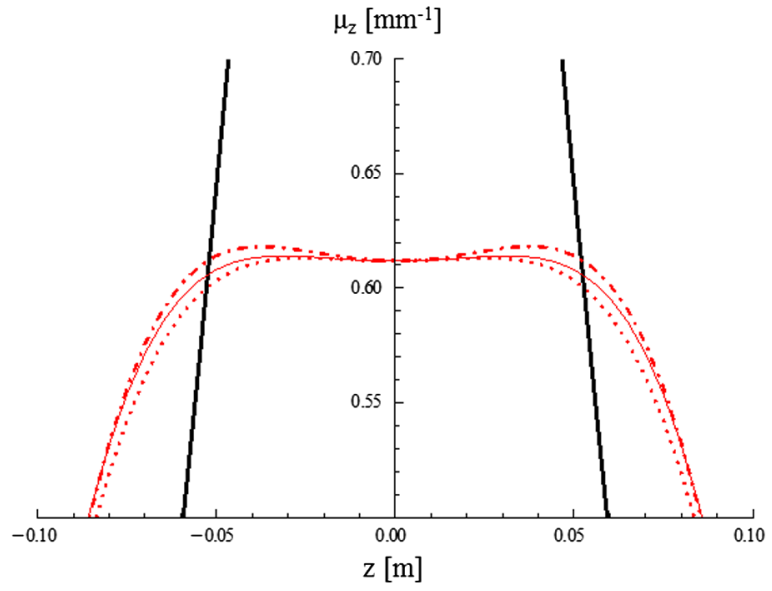

(b)

FIG. 5. Line PU density [mm ${ }^{-1}$ ] vs $z$ [m] (a) normalized to $\mu_{\text {tot }}=135\left(\mathcal{L}_{0}=5 \times 10^{34} \mathrm{~cm}^{-2} \mathrm{~s}^{-1}\right)$ for the four HL-LHC configurations described in Table III, with the black solid line corresponding to case a, and the red solid, dotted-dashed, and dotted lines associated to the cases b, c, and d, respectively. The beneficial impact of the crab-kissing scheme (last 3 cases) is very clear compared to the present HL-LHC baseline (first case), and only a zoom (b) can actually help in appreciating the tiny differences between these three cases. 
TABLE III. Peak line pile up density for the HL-LHC beam (worst case at $\mathcal{L}_{0}=5 \times 10^{34} \mathrm{~cm}^{-2} \mathrm{~s}^{-1}$, i.e., $\mu_{\text {tot }}=135$ ) and need in terms of crab-cavity (CC) voltage for a few selected configurations in terms of bunch profile (Gaussian or pseudorectangular), colliding scheme (crab-crossing or crab-kissing), optics (round or flat) and crossing angle (with electromagnetic wires assumed in the fourth case to mitigate the long-range beam-beam interactions at smaller crossing angle).

\begin{tabular}{|c|c|c|c|c|}
\hline Cases & Case a & Case b & Case c & Case d \\
\hline Colliding scheme & Crab-crossing & Crab-kissing & Crab-kissing & Crab-kissing \\
\hline Bunch profile & $\begin{array}{c}\text { Gaussian } \\
\left(\sigma_{z}=7.5 \mathrm{~cm}\right)\end{array}$ & $\begin{array}{l}\text { Pseudo-rectangular } \\
\left(\sigma_{z}=10.0 \mathrm{~cm}\right)\end{array}$ & $\begin{array}{l}\text { Pseudo-rectangular } \\
\quad\left(\sigma_{z}=10.0 \mathrm{~cm}\right)\end{array}$ & $\begin{array}{l}\text { Pseudo-rectangular } \\
\quad\left(\sigma_{z}=10.0 \mathrm{~cm}\right)\end{array}$ \\
\hline $\mathrm{BB}$ wire compensator & No & No & No & Yes \\
\hline Optics & \multicolumn{2}{|c|}{$\beta_{\times}^{*} / \beta_{\|}^{*}=15 / 15 \mathrm{~cm}$} & \multicolumn{2}{|c|}{$\beta_{\times}^{*} / \beta_{\|}^{*}=30 / 10 \mathrm{~cm}$} \\
\hline Full crossing-angle & $590(12.5 \sigma)$ & $590(12.5 \sigma)$ & $500(15.0 \sigma)$ & $370(11.0 \sigma)$ \\
\hline $\mathrm{CC}$ voltage $[\mathrm{MV}]$ in $\times$ plane & 12.0 & 12.0 & 10.2 & 7.5 \\
\hline $\mathrm{CC}$ voltage $[\mathrm{MV}]$ in $\|$ plane & 0 & 11.4 & 8.1 & 8.1 \\
\hline Peak line PU density $\left[\mathrm{mm}^{-1}\right]$ & 1.22 & 0.61 & 0.61 & 0.61 \\
\hline
\end{tabular}

develop and the peak density is no longer reached at the IP but on either side (where the "crab-kiss" is less efficient due to the RF curvature of the crab-cavity deflecting field but also due to the hour-glass effect which increases the beam size at the head and tail of the bunch).

With four crab-cavities per beam per IR side as recently integrated into the HL-LHC layout, and an engineering specification of 3.3 MV per cavity but with a good hope that 4.0-5.0 MV remains within reach [18], the CK scheme can presently be fully exploited only with flat optics (see Table III), with lets say $2 \mathrm{H} \times 2 \mathrm{~V}$ crab-cavities installed per beam per IR side, and the most performant cavities "oriented" according to the crossing plane. This situation is however not ideal in terms of RF margin (except in the fourth case of Table III with flat optics and beam-beam wires), nor in terms of backward compatibility with the present baseline, i.e., preserving head-on collisions till a crossing angle of $\sim 600 \mu \mathrm{rad}$. Hence optics and layout design studies have been relaunched to make the crabcavities more efficient, by increasing the $\beta$-functions at their respective location, but with possible impacts on impedance mitigation needs, and also field quality and machine protection to be reassessed with twice less cavities in a given transverse plane but at increased $\beta$-functions (see, e.g., $[19,20]$ where these aspects are discussed for the present baseline configuration).

\section{LEVELING TECHNIQUES AND PERFORMANCE EVALUATION OF THE VARIOUS SCHEMES}

The aim of this section is to analyze the CK scheme in terms of leveling concept and, ultimately, performance reach. Profiles will be given for various quantities during the store, including of course the integrated performance. The discussion will be limited to the two HL-LHC configurations described by the cases a and $\mathrm{c}$ of Table III, i.e., the HL-LHC baseline with round optics, crab-crossing and luminosity leveling with $\beta^{*}$, and a variant with flat optics, assuming new hardware for the implementation the CK scheme (but not necessarily including beam-beam wires), and a new leveling technique which is detailed hereafter.

\section{A. Leveling}

A certain number of leveling techniques have been proposed so far and analyzed for the HL-LHC. Each of them presents however some drawbacks, or even clear weaknesses, which can be of very different nature, namely: (i) a poor dynamic range in the case of luminosity leveling with the bunch length, (ii) possible life time and/or instability problems in the case of luminosity leveling with the parallel separation at the IP (still to be confirmed during the LHC Run II), (iii) a line PU density well beyond limits (up to 5 events $/ \mathrm{mm}$ ) but a strongly reduced head-on beambeam tune shift in the case of luminosity leveling with the crabbing angle at minimum $\beta^{*}$, (iv) and conversely a more reasonable luminous region but a maximized beam-beam tune shift (up to $\Delta Q_{b b} \approx 0.03$ at the beginning of the store, including head-on collisions in LHCb) in the case of luminosity leveling with $\beta^{*}$ with full crabbing angle (see Fig. 6). According to the LHC Run I experience and a beam-beam limit probably sitting at or beyond $\Delta Q_{b b} \approx$ 0.03 as demonstrated in dedicated machine experiments $[21,22]$, the last option (iv) has then been retained for the present HL-LHC baseline, at least for leveling the luminosity delivered to the ATLAS and CMS experiments. In all cases, however, none of the above leveling techniques is able to act coherently both on the luminosity, the line PU density and the head-on beam-beam tune shift, simply because none of them is based on a reduction of the collision time. This point really marks the conceptual difference of the $\mathrm{CK}$ scheme with respect to any other luminosity leveling knobs proposed so far.

In the CK scheme, three well-distinct leveling periods can actually be distinguished during the store. During the first period, both the luminosity and the peak line PU density can be leveled to a prescribed value, e.g., $\mathcal{L}_{0}=$ $5 \times 10^{34} \mathrm{~cm}^{-2} \mathrm{~s}^{-1}$ and $\hat{\mu}_{z}=0.61$ event $/ \mathrm{mm}$, respectively, 


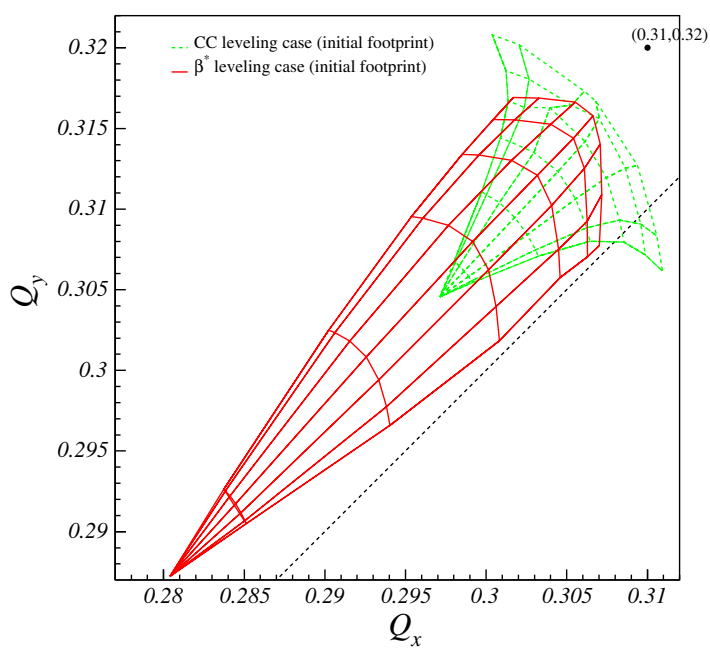

(a)

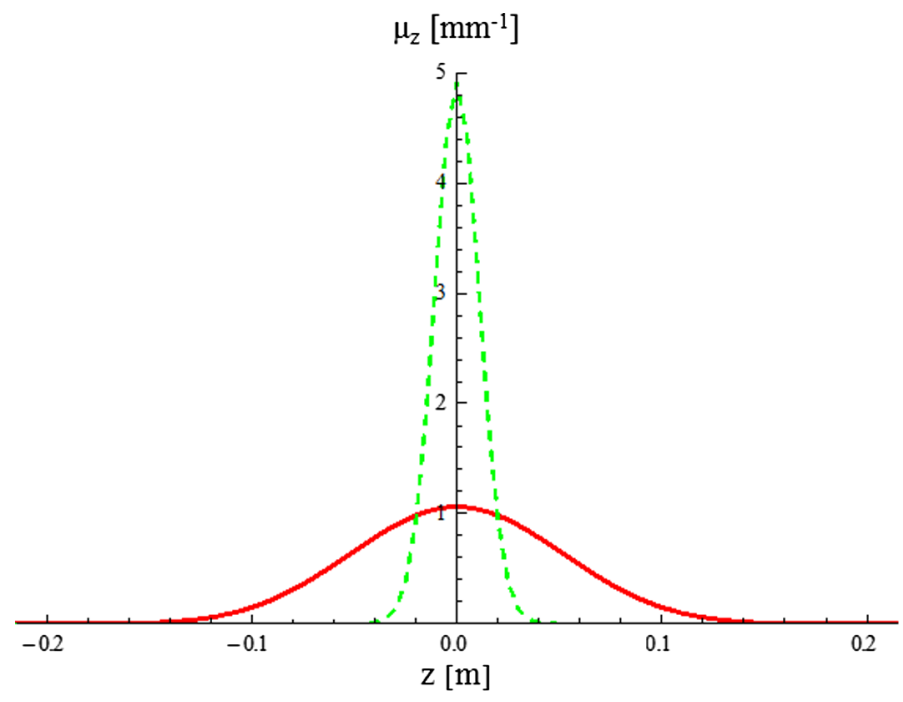

(b)

FIG. 6. Leveling the HL-LHC luminosity by acting either on the crabbing angle (dashed line) at constant (minimum) $\beta^{*}$, or on $\beta^{*}$ itself (solid lines) at constant (full) crabbing angle: main pros and cons in terms of line pile up density (b) and beam-beam effects [as illustrated in (a) for the corresponding tune footprints up to $6 \sigma$ betatron amplitude]. The line PU density is in particular well beyond the limits for the first option, of the order of $\hat{\mu}_{z} \approx 5$ events $/ \mathrm{mm}$ at the beginning of the store, although the situation would be very favorable in terms of beam-beam tune shift. The beam-beam tune shift is on the contrary maximized for the second option, reaching up to $\Delta Q_{b b} \approx 0.03$ at full current and assuming three insertions providing head-on or nearly head-on collisions (i.e., including the contribution of LHCb not equipped with crab-cavities but running at rather large $\beta^{*}$ and therefore rather small Piwinsky angle).

by acting individually onto two machine parameters. The first knob is the kissing angle (i.e., the voltage of the crabcavities acting in the separation plane) set to a nonzero value in order to reach the specified peak PU density. The luminosity is then already reduced with respect to its virtual value (together with the beam-beam tune shift). If the luminosity, i.e., the total number $\mu_{\mathrm{tot}}$ of pile up events, is still too high with respect to its prescribed value, the second $\mathrm{knob}$ to be used is then either $\beta^{*}$ or the crabbing angle, and possibly playing on the first and second knob iteratively in order to match simultaneously the two constraints on $\mu_{\text {tot }}$ and $\hat{\mu}_{z}$. In all the rest of the paper we will limit our discussion to the case where $\beta^{*}$ is kept constant, set to its minimum during the $\mathrm{CK}$ leveling process. This means that the leveling will be achieved exclusively by using the crabbing and the kissing angles, $\alpha_{\times}$and $\alpha_{\|}$, which also turn out to be two orthogonal knobs for the two quantities to level. In this configuration, the first leveling period ends up when head-on collisions are reestablished in the crossing plane $\left(\alpha_{\times} \equiv \theta_{\times}\right)$, and the kissing angle has already been reduced accordingly in order to keep matched the two constraints on $\mu_{\mathrm{tot}}$ and $\hat{\mu}_{z}$ despite of the proton burn off. A second period then starts, where the kissing angle continues to go down, but cannot be reduced as fast as it should be to keep the luminosity constant, unless violating the constraint imposed on the peak line PU density. Hence, the luminosity starts to decay during this second period, but not as fast as a natural decay at constant machine parameters. This second period ends up when the kissing angle reaches zero, which means that head-on collisions are reestablished as well in the parallel separation plane, and the third period starts with the natural decay of the luminosity.

Considering three possible HL-LHC configurations, without or with CK scheme (cases a and c of Table III), and extending the second case to a third case where the instantaneous luminosity could be pushed for $1-2 \mathrm{~h}$ up to $\mathcal{L}_{0}=7.0-7.5 \times 10^{34} \mathrm{~cm}^{-2} \mathrm{~s}^{-1}$ (i.e., $\mu_{\mathrm{tot}} \approx 180-200$ ) at $\hat{\mu}_{z}=1$ event $/ \mathrm{mm}$, the time evolution of the most relevant quantities are showed in Fig. 7, namely: $\beta^{*}$ and the voltage of the crab-cavities in the two transverse planes, together with the corresponding instantaneous luminosity and the peak line PU density in stable beam. The corresponding integrated performance profiles are then reported in Fig. 8. At constant $\mu_{\text {tot }}$, the integrated luminosity delivered by the CK scheme is slightly smaller. However, after $6-8 \mathrm{~h}$ of stable beam, which corresponds to the average fill duration targeted by the HLLHC, this performance loss hardly exceeds a few percents, which is certainly marginal compared to the benefits in terms of quality of the delivered data (a factor of 2 gained in peak line PU density) and therefore useful luminosity. In an optimization process between the machine and the experiment detector, it is then legitimate to think that a certain fraction of this gain in data quality could be redistributed to further push the instantaneous luminosity, e.g., up to $\mathcal{L}_{0}=7-7.5 \times 10^{34} \mathrm{~cm}^{-2} \mathrm{~s}^{-1}$, and therefore further improve in the end the integrated performance of the machine by 20\%-30\% (see Fig. 8) at constant or even still slightly improved data quality $\left(\hat{\mu}_{z} \lesssim 1 \mathrm{event} / \mathrm{mm}\right)$. 


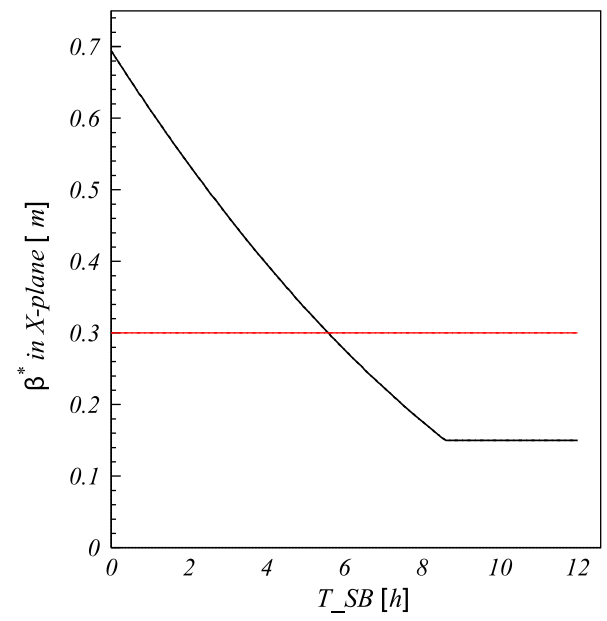

(a)

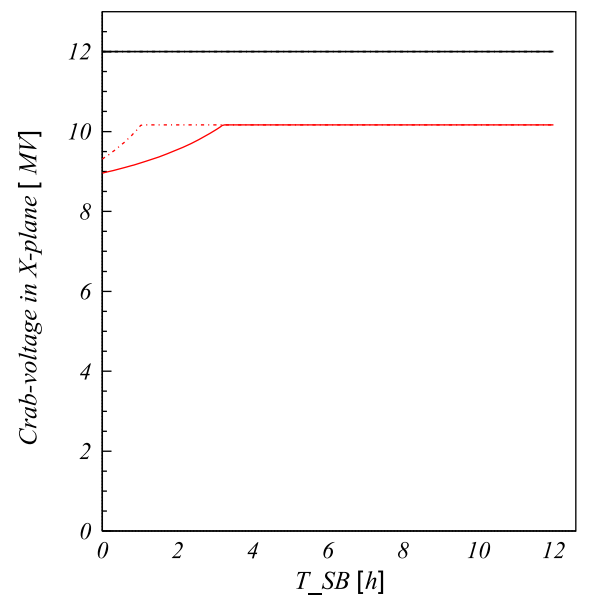

(c)

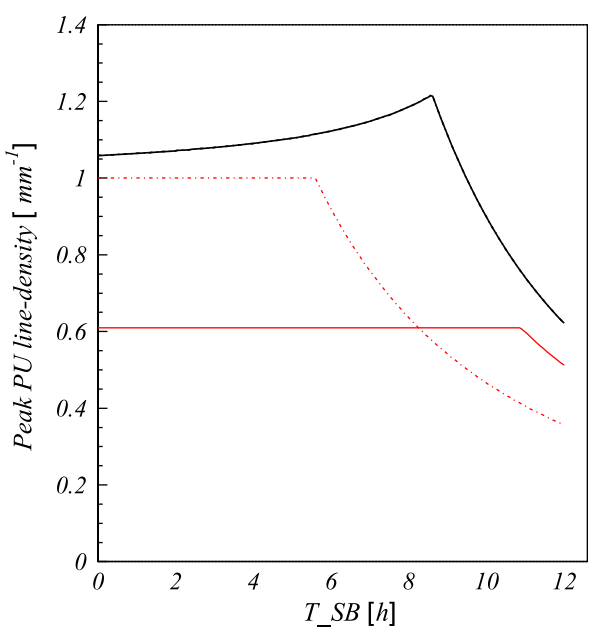

(e)

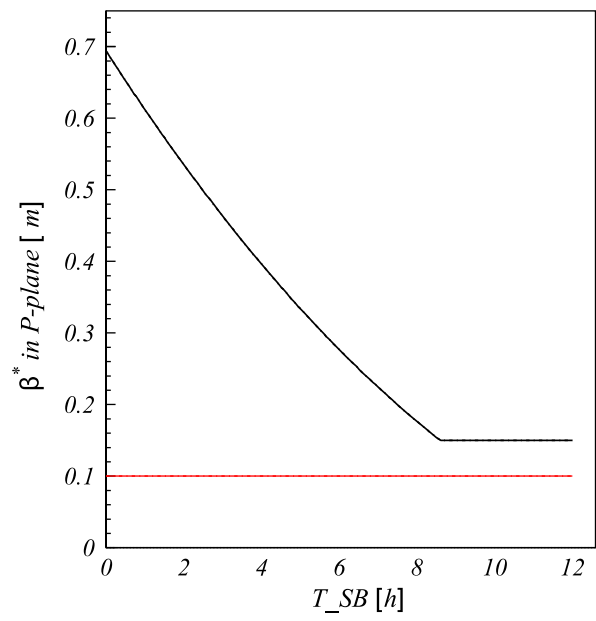

(b)

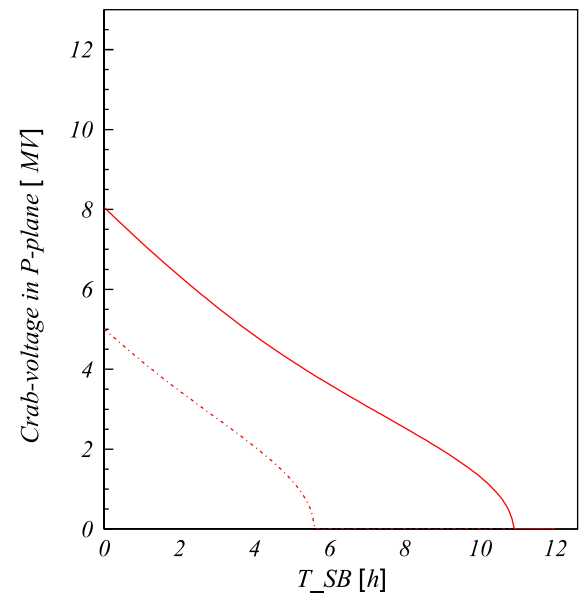

(d)

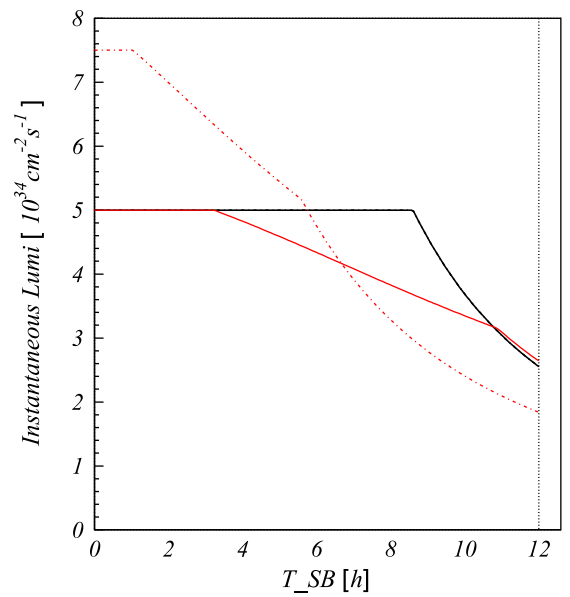

(f)

FIG. 7. Evolution of $\beta^{*}(\mathrm{a}-\mathrm{b})$ and crab-cavity voltage program (c-d) in stable beam (SB), and resulting profiles for the peak line PU density (e) and the instantaneous luminosity (f), for three possible HL-LHC running scenarios, namely: the baseline $\beta^{*}$ leveling scenario (black solid lines) and a first crab-kissing scenario (red solid lines) corresponding to the cases a and c of Table III, respectively, and the same CK configuration but where the luminosity and the peak PU density are pushed up to $\mathcal{L}_{0} \leq 7.5 \times 10^{34} \mathrm{~cm}^{-2} \mathrm{~s}^{-1}\left(\mu_{\mathrm{tot}}=200\right)$ and $\hat{\mu}_{z} \leq 1.0 \mathrm{~mm}^{-1}$ (red dotted-dashed lines). Any possible source of emittance growth is neglected in the above calculations. The beam current decay is assumed to be dominated by the proton burn off due to the collisions in the two high luminosity insertions, assuming a total hadron cross section of $\sigma_{\text {tot }}=100 \mathrm{mb}$ per insertion. 


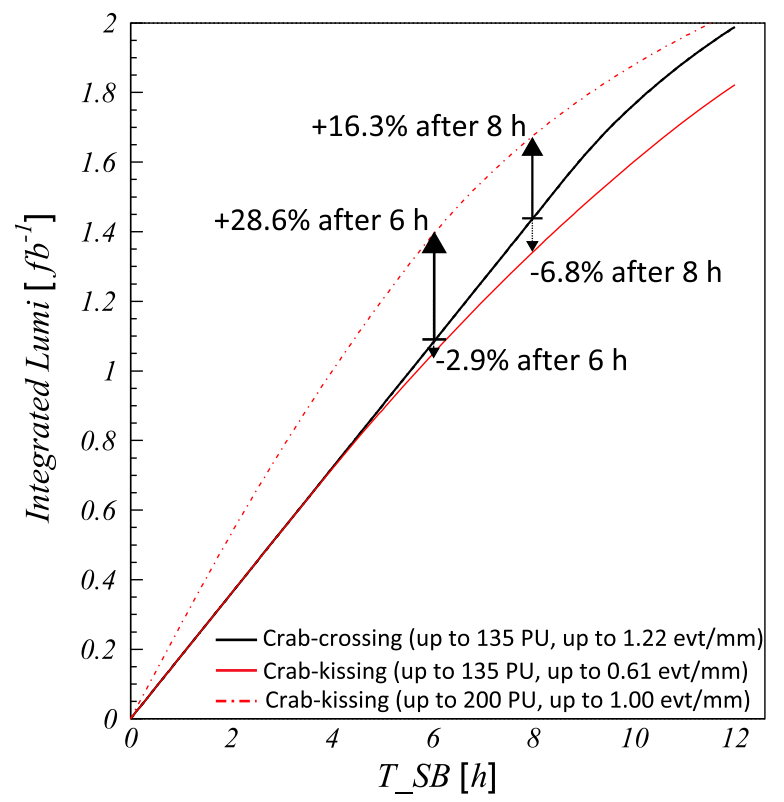

FIG. 8. Performance profile in stable beam for the three HL-LHC configurations discussed in Fig. 7.

\section{SUMMARY AND CONCLUSIONS}

This paper proposes a novel colliding scheme, the socalled crab-kissing (CK) scheme. Relying on crab-cavities acting on the beam in the two transverse planes, the CK scheme opens new possibilities for controlling both the quantity and the quality of the physics data which will be delivered at the HL-LHC, i.e., the total number of interactions per bunch crossing (pile up) together with their line density along the luminous region. A first evaluation of the $\mathrm{CK}$ scheme in terms of beam-beam effects has also demonstrated its viability [23].

With this unexpected connection between pile up density and crab-cavities, these challenging RF devices are and remain more than ever a keystone of the HL-LHC project. Although the crab-kissing concept has now reached a sufficient maturity, and first simulations of the new detector have already confirmed its benefits in terms of primary vertex reconstruction efficiency [24], the present optics and layout version of the HL-LHC is not yet sufficiently optimized for proposing a backward compatible implementation of the CK scheme, with namely: $2 \mathrm{H} \times 2 \mathrm{~V}$ crab-cavities per beam per IP side, both in the ATLAS and CMS experimental insertions, instead of, e.g., $4 \mathrm{H}$ cavities for ATLAS with horizontal crossing and conversely for CMS with vertical crossing. Indeed such modifications would be optimal to fully exploit the CK scheme with flat optics, but definitely counterproductive to maximize the integrated performance of the baseline HL-LHC round optics. This difficulty is not strictly speaking a show stopper to the scheme, but maximizing the performance of flat optics at the price of downgrading the potential of round optics presents obviously a certain level of risk that cannot be overlooked at this stage of the project. This is the main reason why conceptual work has been relaunched to reoptimize accordingly the optics and layout of the HLLHC, basically to increase at constant $\beta^{*}$ the $\beta$-functions at the crab-cavity location and therefore make them more efficient at constant voltage, but of course with pros and cons which will have to be carefully evaluated for a sound implementation of the CK scheme into the HL-LHC baseline.

\section{ACKNOWLEDGMENTS}

I would like to thank L. Rossi for asking me to look into this aspect of leveling and pile up density, at the extreme branching point between machine and experiments. B. Di Girolamo and A. Ball also played a very important role for the fruitful exchanges we had and which certainly motivated me in elaborating the crab-kissing proposal. A. Valishev has been a key player, contributing very early to this proposal with its validation in terms of beam-beam effects. This aspect will form the subject of a separate paper. Finally, I would like to thank O. Brüning for having supported this work in his group, but also and mainly for having suggested me, since the very beginning, to investigate possible benefits of non-Gaussian bunch profiles. Without this request, I would not have developed the required analytical tools, which enlarged my vision, and enabled the emergence of this new idea. Finally I would like to express my gratitude to M. Giovannozzi for the accurate and patient work of proofreading and commenting on the draft manuscript of this article. The HiLumi LHC Design Study [25] is included in the High Luminosity LHC project and is partly funded by the European Commission within the Framework Programme 7 Capacities Specific Programme, Grant Agreement No. 284404.

[1] L. Rossi, in Proceedings of the 2nd International Particle Accelerator Conference, San Sebastián, Spain (EPS-AG, Spain, 2011), p. 908.

[2] S. Fartoukh, Phys. Rev. ST Accel. Beams 16, 111002 (2013).

[3] H. Damerau, R. Garoby, S. Gilardoni, B. Goddard, K. Hanke, A. Lombardi, M. Meddahi, B. Mikulec, E. Shaposhnikova, and M. Vretenar, in Proceedings of the 3rd International Particle Accelerator Conference, New Orleans, LA, 2012 (IEEE, Piscataway, NJ, 2012).

[4] H. Bartosik, T. Argyropoulos, B. Goddard, G. Iadarola, Y. Papaphilippou, G. Rumolo, and E. Shaposhnikova, in The Review of the LHC and Injector Upgrade Plans (RLIUP), Archamps, France, 2013, https://indico.cern.ch/event/ 260492/.

[5] S. Fartoukh, R. De Maria, in Proceedings of the 3rd International Particle Accelerator Conference, New Orleans, LA, 2012 (IEEE, Piscataway, NJ, 2012), p. 145.

[6] E. Todesco, H. Allain, G. Ambrosio, G. Arduini, F. Cerutti, R. De Maria, L. Esposito, S. Fartoukh, P. Ferracin, 
H. Felice, R. Gupta, R. Kersevan, N. Mokhov, T. Nakamoto, I. Rakno, J. M. Rifflet, L. Rossi, G. L. Sabbi, M. Segreti, F. Toral, Q. Xu, P. Wanderer, and R. van Weelderen, IEEE Trans. Appl. Supercond. 24 (2014); also in CERN Report No. ATS 2014-0036, 2014.

[7] R. B. Palmer, eConf C8806271 (1988), 613; also available in Report No. SLAC-PUB-4707.

[8] R. Calaga, R. Tomas, and F. Zimmermann, in Proceedings of the 3rd CARE-HHH-APD Workshop: Towards a Roadmap for the Upgrade of the LHC and GSI Accelerator Complex, Valencia, Spain, 2006, p. 77, Report No. CERN2007-02, CERN, Geneva, Switzerland.

[9] https://espace.cern.ch/HiLumi/PLC/default.aspx.

[10] O. Brüning, P. Collier, P. Lebrun, S. Myers, R. Ostojic, J. Pool, and P. Proudlock, CERN Report No. CERN-2004003-V-1, 2004.

[11] J. P. Koutchouk, CERN Report No. LHC-Project-Note 223, 2000.

[12] F. Zimmermann, P. Lebrun, T. Sen, V. Shiltsev, and X. L. Zhang, CERN Report No. AB-Note-2004-041, 2004.

[13] C. Milardi, D. Alesini, M. A. Preger, P. Raimondi, D. Shatilov, and M. Zobov, in Proceedings of CARE-HHHAPD IR'07 Workshop, Frascati, 2007, p. 92, Report No. CERN-2008-006, CERN, Geneva, Switzerland; arXiv:0803.1544.

[14] U. Dorda, J. Koutchouk, R. Tomas, J. Wenninger, F. Zimmermann, R. Calaga, and W. Fischer, in Proceedings of the 11th European Particle Accelerator Conference, Genoa, 2008 (EPS-AG, Genoa, Italy, 2008), p. 3176.

[15] R. Calaga, W. Fischer, G. Robert-Demolaize, and N. Milas, Phys. Rev. ST Accel. Beams 14, 091001 (2011).

[16] F. Zimmerman and O. Brüning, in Proceedings of the 3rd International Particle Accelerator Conference, New Orleans, LA, 2012 (IEEE, Piscataway, NJ, 2012).
[17] R. Tomas et al., in Proceedings of the Review of the LHC and Injectors Upgrade Plans Workshop (RLIUP), Archamps, France, 2013 (to be published); also available in Report No. CERN-ACC-NOTE-2014-074, CERN, Geneva, Switzerland.

[18] P. Baudrenghien, K. Brodzinski, R. Calaga, O. Capatina, E. Jensen, A. Macpherson, E. Montesinos, and V. Parma, CERN, Report No. CERN-ACC-NOTE-2013-003, 2013.

[19] J. Barranco Garcia, R. Calaga, R. De Maria, M. Giovannozzi, A. Grudiev, and R. Tomas, in Proceedings of the 3rd International Particle Accelerator Conference, New Orleans, LA, 2012 (IEEE, Piscataway, NJ, 2012), p. 1873.

[20] T. Baer, J. Barranco Garca, R. Calaga, R. Tomas, J. Wenninger, B. Yee, and F. Zimmermann, in Proceedings of the 24th Particle Accelerator Conference, PAC-2011, New York, 2011 (IEEE, New York, 2011), p. 76.

[21] R. Alemany, X. Buffat, R. Calaga, K. Cornelis, M. Fitterer, R. Giachino, W. Herr, A. McPherson, R. Miyamoto, G. Papotti, T. Pieloni, S. Redaelli, F. Roncarolo, M. Schaumann, R. Suykerbuyk, G. Trad, and S. Paret, CERN Report No. CERN-ATS-Note-2011-029-MD, 2011.

[22] X. Buffat, R. Alemany-Fernandez, R. Giachino, W. Herr, G. Papotti, T. Pieloni, R. Calaga, S. M. White, and M. Schaumann, CERN Report No. CERN-ATS-2011-151, 2011.

[23] A. Valishev, S. Fartoukh, and D. Shatilov, "Beam-Beam Effects with the Crab-Kissing Scheme" (to be published).

[24] F. Meloni and S. Pagan Griso (private communication); ATLAS internal report (to be published); See also A. Ball, B. Di Girolamo, S. Fartoukh, and L. Rossi, 3rd Joint HiLumi LHC-LARP Annual Meeting, Daresbury, 11-14 November 2013, Daresbury, UK, https://indico.cern.ch/ conferenceDisplay.py? confId=257368.

[25] http://hilumilhc.web.cern.ch. 